\title{
Sex-specific transcriptomic and epitranscriptomic signatures of PTSD-like fear acquisition.
}

Andre Martins Reis ${ }^{1,2}$, Jillian Hammond ${ }^{1,2}$, Igor Stevanovski ${ }^{1,2}$, Jonathon C Arnold ${ }^{3,4,5}$, lain S. McGregor ${ }^{4,5,6}$, Ira Deveson ${ }^{1,2^{*}}$, Anand Gururajan ${ }^{4,6^{*}}$.

${ }^{1}$ Kinghorn Centre for Clinical Genomics, Garvan Institute of Medical Research, NSW, Australia.

${ }^{2}$ St Vincent's Clinical School, University of New South Wales, NSW, Australia.

${ }^{3}$ Faculty of Medicine and Health, Discipline of Pharmacology, The University of Sydney, NSW, Australia.

${ }^{4}$ Brain \& Mind Centre, The University of Sydney, NSW, Australia.

${ }^{5}$ The Lambert Initiative for Cannabinoid Therapeutics, Australia

${ }^{6}$ School of Psychology, Faculty of Science, The University of Sydney, NSW, Australia

*Co-last authors

Corresponding Author: Anand Gururajan, anand.gururajan@sydney.edu.au 


\begin{abstract}
Our understanding of the molecular pathology of posttraumatic stress disorder (PTSD) is rapidly evolving and is being driven by advances in sequencing techniques. Conventional short-read RNA sequencing (RNA-seq) is a central tool in transcriptomics research that enables unbiased gene expression profiling. With the recent emergence of Oxford Nanopore direct RNA-seq (dRNA-seq), it is now also possible to interrogate diverse RNA modifications, collectively known as the 'epitranscriptome'. Here, we present our analyses of the male and female mouse amygdala transcriptome and epitranscriptome, obtained using parallel Illumina RNA-seq and Oxford Nanopore dRNA-seq, associated with the acquisition of PTSDlike fear induced by Pavlovian cued-fear conditioning. We report significant sex-specific differences in the amygdala transcriptional response during fear acquisition, and a range of shared and dimorphic epitranscriptomic signatures. Differential RNA modifications are enriched among mRNA transcripts associated with neurotransmitter regulation and mitochondrial function, many of which have been previously implicated in PTSD. Very few differentially modified transcripts are also differentially expressed, suggesting an influential, expression-independent role for epitranscriptional regulation in PTSD-like fear-acquisition. Overall, our application of conventional and newly developed methods provides a platform for future work that will lead to new insights into and therapeutics for PTSD.
\end{abstract}




\section{Main text}

The psychology of posttraumatic stress disorder (PTSD) is rooted in the acquisition of memories associated with specific traumatic events ${ }^{1}$. Neuromolecular changes in brain structures such as the amygdala, which play critical roles in learning and memory, have been implicated in the pathophysiology of PTSD². Several studies have identified the impact of PTSD trauma on the epigenome ${ }^{3,4}$. However, over the last decade, there is increasing evidence implicating RNA modifications, collectively known as the 'epitranscriptome', in brain function and behaviour ${ }^{5-8}$. These modifications can impact RNA stability, localisation and regulate translation in a dynamic and reversible manner. As such, they have the potential to rapidly fine-tune responses to external stimuli and may underpin aspects of fear learning and memory.

Here we report the results of our study that focused on capturing changes in the amygdala transcriptome and epitranscriptome during the acquisition of the conditioned fear response. We leveraged parallel short-read RNA-seq (Illumina NextSeq) and long-read direct RNA-seq (dRNA-seq; Oxford Nanopore Technology PromethION) to profile gene expression and RNA modifications, respectively, in a mouse model for aspects of PTSD.

Our PTSD mouse model was developed using a modified auditory, cued-fear conditioning protocol developed by Siegmund and Wotjak $^{9}$ and has been validated for its face, construct and predictive validities ${ }^{10-14}$. Epidemiological evidence suggests that females are at higher risk (2:1) of developing PTSD following trauma ${ }^{15}$. However, to our knowledge, few PTSD-stress paradigms, including the one we have employed here, have been applied in females ${ }^{16}$. Accordingly, for our first experiment, we investigated if cued-fear memory was observable in adult female and male mice $24 \mathrm{~h}$ after fear conditioning (Figure 1a). We observed a significant increase in freezing behaviour in both male and female conditioned (shocked) mice compared to non-conditioned (non-shocked) controls, with females freezing significantly more than males (Figure 1b). Our findings were consistent with one other study which also reported greater cued fear expression in females than males, suggestive of sex-differences in discriminative ability to the cue $^{17}$.

These findings led us to investigate whether this significant sex-difference in expression of PTSD-like fear memory could be associated with differences in transcriptional and epitranscriptional processes associated with the initial acquisition of the fear memory. To address this, a separate group of mice was sacrificed within 15 min of the fear conditioning procedure, capturing the earliest molecular signatures of fear acquisition. Messenger RNA (mRNA) was extracted and isolated from the amygdala of each individual, pooled in groups of 3-4 individuals, and analysed by short-read RNA-seq to assess differential gene expression (DGE) responses to the PTSD-like stressor (Figure 1a, Supplementary Tables 1-3). We obtained on average 37.9 million read pairs per sample $\left(S D=7.23 \times 10^{6}\right.$; Supplementary Table 
1). In each library, most of the reads were successfully mapped to the reference genome $(84.72 \% \pm 0.56)$ and subsequently assigned to an annotated gene $(65.83 \% \pm 1.03$; Supplementary Table 1).

We identified 190 up-regulated and 401 down-regulated genes in conditioned male mice (fold-change $>0.2$ and FDR $<0.05$; Figure 1d, Supplementary Table 2). The expression of the top 10 DGEs in males included Zinc finger and BTB domain containing 16 (Zbtb16), LIM homeobox protein 9 (Lhx9), fibrinogen C domain containing 1 (Fibcd1), serum/glucocorticoid regulated kinase 1 (Sgk1), and IsI1 transcription factor, LIM/homeodomain (Is/1), all of which were $\mathrm{qPCR}$ validated using the same samples that were sequenced (Figure 1e, $\mathbf{f}$ ).

Zbtb16 was found to be significantly upregulated in conditioned male mice and is a transcriptional regulator involved in a myriad of processes including those linked to neurodevelopment ${ }^{18,19}$. Zbtb16 knockout mice show several behavioural impairments relevant to neurodevelopmental disorders such as autism spectrum disorder and schizophrenia. In addition to impaired cortical morphology, these mice also show dysregulation in genes associated with myelination and neurogenesis ${ }^{20}$. Functional expression of this gene in the adult hypothalamus has been recently linked to regulation of metabolism ${ }^{21}$. Lhx9 and $I s / 1$ are transcription factors which also have roles in neurodevelopment ${ }^{22}$ 23. Fibcd1 belongs to a class of proteins known as fibrinogen-related domains (FreD) with multiple functions which include innate immunity and are expressed in glial cells in the human brain ${ }^{24}$. To our knowledge the functional expression of these four genes in the adult amygdala and in response to PTSD paradigms such as the one used in our study has heretofore not been characterised. They therefore represent interesting targets for future investigations.

Sgk1 was downregulated in expression. It is involved in several functions which include regulating ion channel activity, interfering with transcription and neuronal excitability ${ }^{25}$. In the amygdala, an earlier microarray study reported an increase in Sgk1 expression following cued-fear conditioning which contrasts with our findings; however, these experiments were carried out using a different conditioning protocol and analysis of amygdala RNA was at later time points ${ }^{26}$. It is worth noting that $S g k 1$ is also expressed in other brain regions including the hippocampus, where it has a role in contextual fear memory formation ${ }^{27}$, and in the prefrontal cortex (PFC). A downregulation in expression has been reported in post-mortem tissue analyses of PFC tissue from patients who had suffered from PTSD, and is associated with increased expression of contextual fear memory in rats ${ }^{28}$.

Independent Gene Set Enrichment Analysis (GSEA) for conditioned male mice indicated a significant over-representation of genes implicated in synaptic activity (Supplementary Table 3), a process that is well-known to be implicated in the acquisition of memories ${ }^{29-32}$. 
To our surprise, despite the increased expression of cued-fear memory, fear conditioning induced a modest transcriptional response in conditioned female mice (Supplementary Table 4). We observed a significant decrease in expression for only a single gene, Rps27A; however, this was not validated using qPCR. There remains debate as to whether the amygdala is a sexually dimorphic structure ${ }^{33}$. Evidence suggests there are intrinsic differences linked to sex hormones that may explain our sex-specific observations in the formation and expression of emotional memories ${ }^{34-37}$. Our findings contrast with several studies which have examined sex-differences in PTSD-like phenotypes, albeit in rats, with different inducing paradigms. Acute predator scent stress (PSS) induced significant differential expression of amygdala genes in males and female rats, detected using a microarray screen, that were classified as having an extreme behavioural response ${ }^{38}$. A subsequent re-analysis of this data revealed sexdifferences in cellular composition of the amygdala but no effect of PSS. Controlling for cell subtype revealed shared as well as dimorphic gene expression profiles and enrichment analyses in the amygdala associated with $\mathrm{EBR}^{39}$. Using a single-prolonged stress (SPS) which consisted of $2 \mathrm{~h}$ restraint stress, $20 \mathrm{~min}$ forced swim followed by brief exposure to diethyl ether or exposure to a live predator, male rats exhibited a significantly higher acoustic startle, and increased sensitivity to dexamethasone-induced suppression of the HPA-axis response compared to females. SPS decreased social interaction and sucrose preference females ${ }^{40}$. Gonadal hormone status of males had no effect on SPS-induced startle hyperreactivity or dexamethasone responses but did influence female sucrose preference. Analysis of the right basolateral amygdala revealed a paradoxical increase in c-fos expression only in females ${ }^{41}$. It is worth noting that for all the above studies, the transcriptional analyses was done on brain tissue samples collected from 8 days to 2 weeks after the exposure to the traumatic stimuli. There have been several other studies which have examined changes to the transcriptional architecture in the brain in female rodents following chronic adult and early life stress exposure $e^{42-45}$. Our understanding of the immediate changes to the transcriptional architecture in the brain in response to stress in female rodents and how this sets the stage for longer term sequelae is still limited. Overall, our DGE data suggests significant distinctions exist during the early transcriptional events of fear acquisition in males and females warranting much further investigation. 

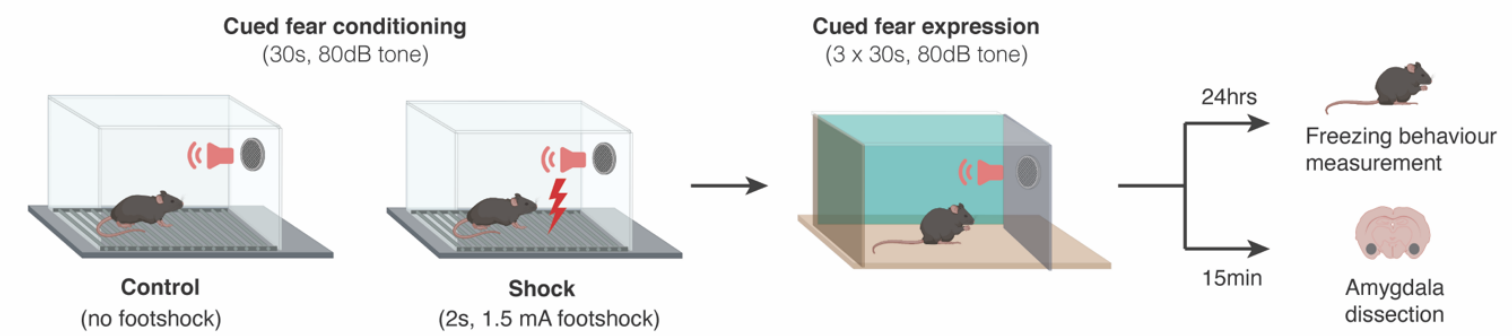

b Experiment layout
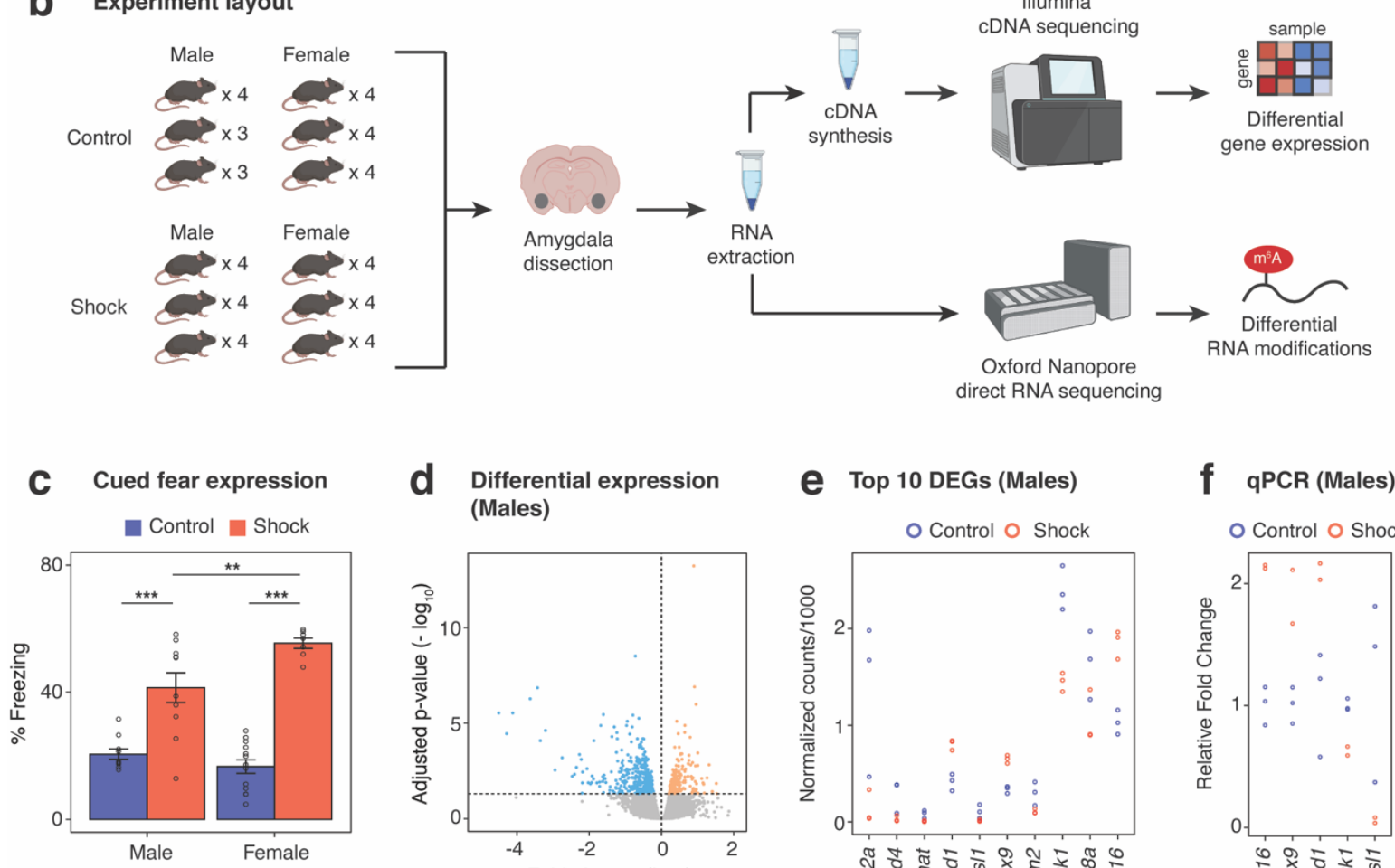
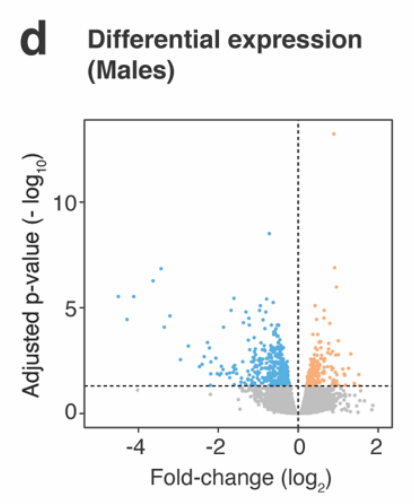

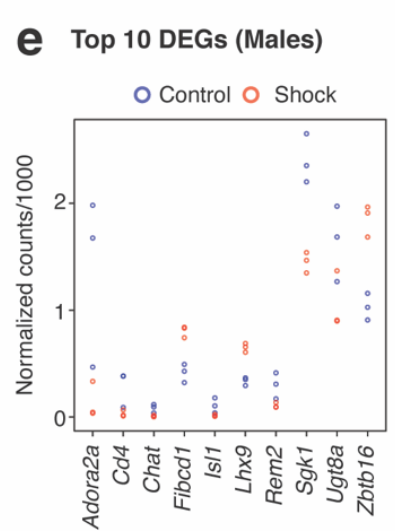

f qPCR (Males)

o Control O Shock

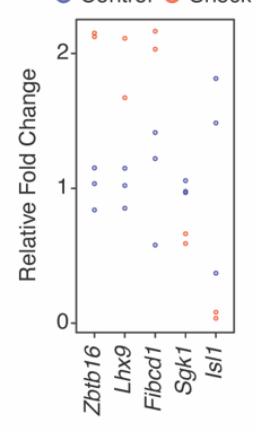

Figure 1. Sex-specific transcriptional profiles are associated with the acquisition of PTSD-like fear memories in the amygdala. (a) Experimental workflow. (b) Adult male and female mice underwent cued-fear conditioning (or the control condition) after which they were tested for cued-fear expression 24hrs later; another group was sacrificed within 15mins of fear conditioning to obtain amygdala RNA which was subsequently pooled for indirect (Illumina) and direct (Oxford Nanopore) sequencing. (c) Face validity of the cued fear conditioning paradigm was verified in male and female mice which showed strong expression of conditioned fear responses. ${ }^{* * *} p<0.001$ relative to non-fear conditioned controls, ${ }^{* *} \mathrm{p}<0.01$ for male vs female conditioned mice. $\mathrm{n}=7$ 10/group. (d) Differential gene expression in the amygdala of male mice; black dots in blue shade represent significantly downregulated genes and black dots in pink shade represent significantly upregulated genes. Thresholds for DESeq2: $|\mathrm{FC}|>20 \%$ and FDR $<0.05$. (e) Top 10 differentially expressed genes in male conditioned mice. (f) qPCR validation for selected differentially expressed genes in males. 
Recent evidence suggests that epitranscriptomic modifications, such as N6-methyladenosine $(\mathrm{m} 6 \mathrm{~A})$, may have widespread regulatory roles in the brain ${ }^{5-8}$. ONT dRNA sequencing has the potential to enable comprehensive profiling of the diverse array of RNA base modifications that together comprise the 'epitranscriptome'46, 47 . However, this is a nascent field and analytical best practices are yet to be established ${ }^{48}$.

To explore epitranscriptome dynamics during fear acquisition, we performed dRNA sequencing on the same amygdala RNA samples as described above. Each sample was sequenced on a single PromethION flow cell, yielding a median of 3.9 million QC-passed reads per sample, each of which represents a native mRNA transcript (Supplementary Table 5). This enabled a broad survey of expressed transcripts (Average read count $>3$ ), with 12,792/32,604 (39.2\%\%) of protein coding genes reaching an average read count greater than 15 , the minimum threshold required for detection of putative RNA modifications (Supplementary Table 6).

An overview of our analysis workflow used to profile RNA modifications is shown in Figure 2a, exemplified using the transcript for Neurensin-2 (Nrsn2), a known modulator of emotional behaviour and putative biomarker for PTSD ${ }^{49}, 50$. Briefly, we used a combination of two recently developed software packages, Xpore ${ }^{51}$ and Nanocompore ${ }^{52}$, to detect differential RNA modifications in the comparison of conditioned vs controls samples. Xpore identified 50,681 candidate modification sites across 9,902 transcripts for males and 37,998 candidates across 8,979 transcripts for females (Figure 2a,b). Nanocompore identified 43,172 candidate modification sites across 1,547 transcripts for males and 33,216 candidates across 1,482 genes for females (Figure 2a,b). The tools use alternative metrics and statistical approaches to identify modified k-mers and concordant results provide the best evidence of modified sites. To maximise stringency, we retained only those candidates that were identified by both Nanocompore and Xpore and collapsed any candidates within $10 \mathrm{nt}$ into a single modification site (Figure 2a). For males, this approach identified 7,487 candidate modifications between conditioned vs control conditions that were collapsed into 3,397 unique differentially modified sites (Figure $\mathbf{2 b}$ ). In females, 4,808 candidates were collapsed into 2,331 unique differentially modified sites (Figure $\mathbf{2 b}$ ). These high confidence sites showed mean $\sim 1$.7-fold higher modification rates, as measured by displacement of the current signal, under the stress condition in both males (stress $=0.48$, control $=0.27$ ) and females (stress $=0.49$, control $=$ 0.29; Figure 2c;).

Most high-confidence modified sites were found within 3' untranslated regions (UTR) of mRNA transcripts, with an enrichment close to the boundary with the coding sequence (CDS) observed in both males and females (Figure $\mathbf{2 d}$,e). A smaller proportion of modified sites were found within 5'UTRs, which are generally shorter than 3'UTRs, but 5'UTRs exhibited the highest density of sites per nucleotide compared to the $3^{\prime} U T R$ and CDS in both sexes 
(Supplementary Figure 1a,b). These observations are consistent with positional distributions previously reported for m6A and other known RNA modifications ${ }^{53-55}$.

Although Nanocompore and Xpore can identify differentially modified bases, neither tool determines the specific type of RNA modification at a given site. To further characterise and validate our modification sites, we assessed the enrichment of sequence k-mers consistent with the canonical m6A DRACH motif ${ }^{56}$. We found an enrichment of DRACH motif k-mers within high-confidence modification sites, compared to 10 sets of random non-modified sites, and no enrichment for k-mers of a randomly selected motif (Figure 2f). We also intersected our set of high-confidence modification sites with RMBase; a database of known RNA modifications ${ }^{57}$. We found 506 annotated RMBase modifications overlapping differentially modified sites in males and 421 in females, with almost all of them identified as m6A (Figure 2b). We retrieved the consensus sequence around these m6A sites and consistently observed the DRACH motif for both sexes (Figure $\mathbf{2 g}$ ). Concordance with previously annotated RNA modifications, and adherence to known patterns of transcript position and sequence context confirms the reliability of our catalogue of differentially modified sites during fear acquisition.

Next, we functionally characterised genes and transcripts identified as being differentially modified. Out of 3,397 and 2,331 modified sites detected in males and females (Supplementary Tables 7, 8), respectively, we found 580 common modifications in 186 genes (average = 3.11 sites/gene; Figure $\mathbf{2 h}$; Supplementary Figure $\mathbf{1 c}$ ), suggesting a degree of both shared and sexually dimorphic epitranscriptome dynamics. GSEA for males and females indicated enrichment of equivalent and/or related gene ontology terms (Figure 2i). Within the top 20 most significantly enriched terms, $60 \%$ were found in both males and females (Supplementary Tables 9,10).

Mitochondrial function and neurotransmitter production/secretion were the predominant functional categories identified by GSEA (Figure 2i). The former is particularly interesting in the context of evidence implicating mitochondrial function in the stress response ${ }^{58-60}$ and mitochondrial dysfunction in PTSD pathology ${ }^{61,62}$. Mitochondrial metabolic processes are components of the cell danger response (CDR) which is activated by stressors. Negative feedback mechanisms exist to 'switch off' the CDR once the stress has passed but if they fail, this may contribute to oxidative stress and inflammation ${ }^{63}$. 
bioRxiv preprint doi: https://doi.org/10.1101/2021.11.25.468910; this version posted November 25, 2021. The copyright holder for this preprint (which was not certified by peer review) is the author/funder, who has granted bioRxiv a license to display the preprint in perpetuity. It is made available under aCC-BY-ND 4.0 International license.

a Epitranscriptome analysis workflow

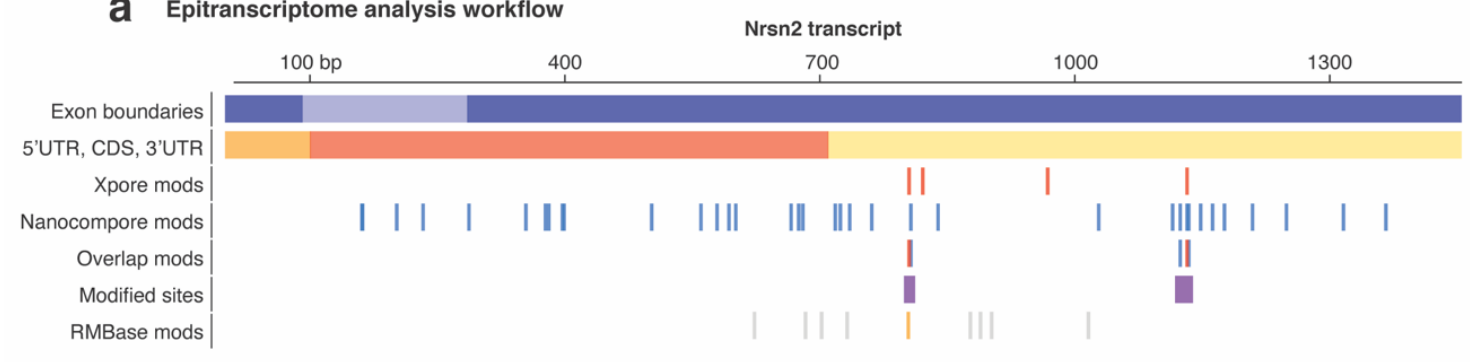

b Detection of RNA mods.
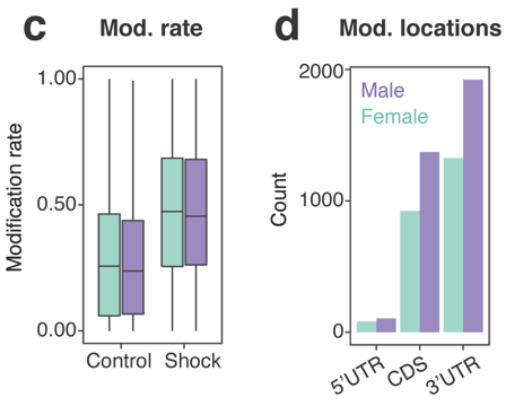

Distribution of mods.
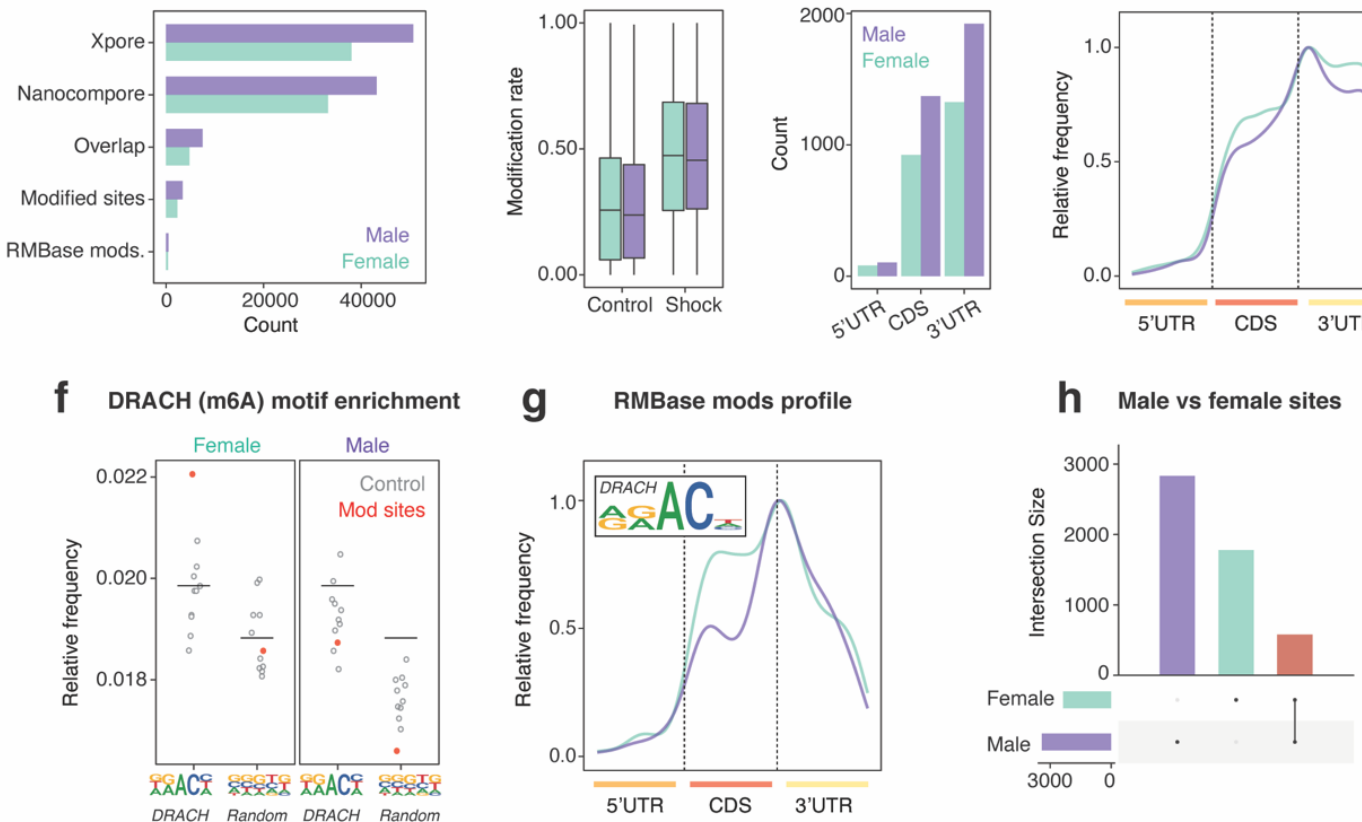

i Top 10 common biological processes in males and females

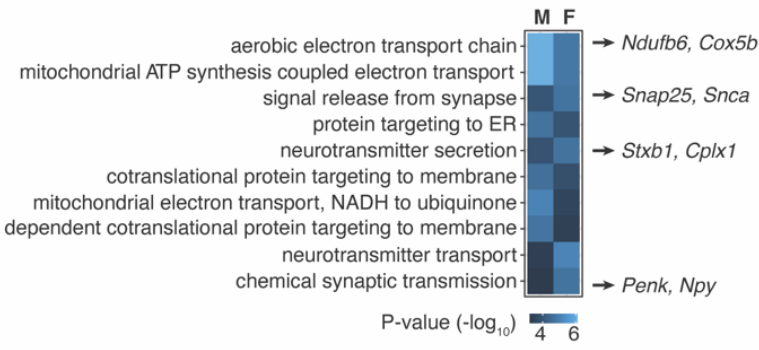

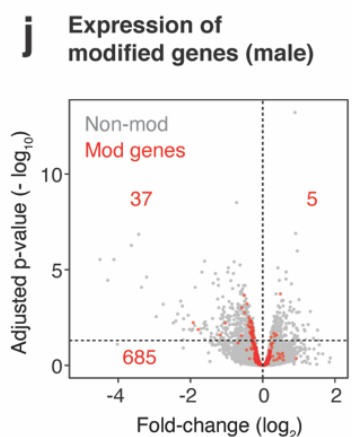

Figure 2. Epitranscriptome signatures of PTSD-like fear acquisition in the amygdala. (a) Schematic example using the Nrsn2 mRNA transcript to illustrate the analytic workflow for profiling RNA modifications in conditioned vs control mice. Briefly, candidate modifications were identified separately by Xpore and Nanocompore. Only overlapping/adjacent candidates identified by both tools were retained and multiple candidates within $10 \mathrm{nt}$ were collapsed into high confidence modified sites. Finally, modified sites were cross-checked against RMBase. (b) The number of candidates and modified sites retained at each step of the workflow just described (c) The modification frequencies recorded by Xpore for modified sites in stress vs control and male vs female samples. (d) The total number of modified sites identified within different regions ( $5^{\prime}$ UTR, CDS and 3'UTR) of mRNA transcripts. (e) The positional distribution of modified sites, averaged across the whole transcriptome, within the same regions. (f) The relative frequency of canonical DRACH motif Kmers vs random k-mers observed within modified sites (blue), as opposed to random sites in matched transcript regions. (g) The positional distribution and k-mer frequency profile of modified sites that also overlapped RMBase modifications, with both showing characteristic features of m6A. (h) The number of modified sites identified that were sex-specific or shared between males and females. (i) The top ten common GO terms enriched among differentially modified genes in males and females. Example genes underlying each term are highlighted. (j) Volcano plot showing gene expression fold-changes observed between stress vs control, with genes that were identified as differentially modified highlighted in red. Minimal overlap between differentially expressed and differentially modified genes is apparent. 
Among the top differentially modified gene candidates were multiple synaptic genes previously implicated in PTSD, including Preproenkaphelin (Penk) ${ }^{64}$ and Neuropeptide $Y$ $(N p y)^{65}$. Evidence suggests the Npy system promotes resilience-to-stress in rodents and reduced NPY in humans is associated with PTSD ${ }^{65}$. We observed differentially modified sites in the 3'UTR and CDS regions of Npy in males and females. In each case, the modification frequency was upregulated during fear conditioning, whereas expression of Npy mRNA was not found to be upregulated, suggesting transcription-independent regulation of Npy by RNA modifications may occur during fear conditioning. The lack of concordance between differential modification and expression in Npy was similarly observed across the transcriptome, with just 150/3,397 (4.41\%) of high confidence modifications being harboured within differentially expressed genes (Figure $\mathbf{2 j}$; Supplementary Table 11). This finding reveals widespread expression-independent epitranscriptomic regulation in the amygdala during fear acquisition and is consistent with previous work showing an absence of correlation between changes in gene expression, translational efficiency, and m6A-methylation ${ }^{66}$. We hypothesise that dynamic RNA modification processes are among the earliest molecular events in fear conditioning and may regulate the activity of key effector genes prior to activation of mechanisms which include but are not limited to differential gene expression in a sex-specific manner.

Gaining an insight into the molecular mechanisms that are triggered immediately following trauma are important to comprehend how they are implicated in the neuropathological cascades that culminate in the onset of PTSD. Our study provides a framework for exploring acquisition-induced transcriptomic and epitranscriptomic events underpinning PTSD-like phenotypes in male and female mice and implicates RNA modifications in the regulation of fear. There is broad scope for future work, but one major issue must be addressed; how does one interrogate the functional roles of RNA modifications ${ }^{67}$ ?

For some modifications such as $\mathrm{m} 6 \mathrm{~A}$, the majority of known m6A sites are unmethylated at baseline ${ }^{68}$. The functional relevance of constitutive versus regulated m6A sites is unknown but such sub-stoichiometric levels would indicate a large margin in which to regulate RNA metabolism and gene expression. Furthermore, while the lifecycle of modifications is thought to be transient, this may vary from transcript to transcript. Our data are also consistent with recent work showing that RNA modifications can form clusters on the same transcript ${ }^{69}$, exerting cumulative or combinatorial effects on RNA metabolism. One low resolution approach to providing answers is by manipulating the expression and activity of epitranscriptomic machinery enzymes - 'writers', 'erasers' and 'readers'. This has been used by numerous studies in the context of learning, memory, and the stress response ${ }^{66,70,71}$. There have also been several pharmacological compounds developed to inhibit or activate these enzymes ${ }^{72,73}$. One higher resolution strategy involves the use of CRISPR-Cas Inspired RNA-targeting (CIRT) system ${ }^{74,75}$ but this is yet to validated in vivo. And lastly, future 
investigations should consider incorporating techniques such as ribosome profiling and proteomics $^{76}$.

In conclusion, our study opens a new and exciting frontier in molecular psychiatry research that has potential to reshape our understanding of stress-induced psychiatric disorders. RNAbased therapies have found success in treatment of diseases from cancer to COVID1977. Perhaps, there is scope to treat psychiatric disorders such as PTSD in a similar manner. 


\section{Acknowledgements}

AG wishes to thank Dr Nicholas Everett and Dr Erin Lynch for technical assistance and the Laboratory Animal Services team at the Brain \& Mind Centre for assistance with animal husbandry. We thank our colleagues Derrick Lin and Warren Kaplan for providing technical support and oversight of high-performance computing at the Garvan Institute. AG also wishes to thank Professor Tim Bredy (University of Queensland) for the many intellectually challenging conversations associated with this study.

\section{Funding}

AG was supported by a University of Sydney Research Fellowship. IWD and AMR are supported by an MRFF Investigator Grant (MRF1173594) to IWD. Animal experiments were conducted at the Brain \& Mind Centre with supplemental financial support provided by the Lambert Initiative. Sequencing experiments were conducted at the Garvan Institute with financial support from the Kinghorn Foundation.

\section{Availability of data and materials}

All data generated or analysed during this study are included in this published article and its supplementary information files. Sequencing files have been uploaded to NCBI GEO (PRJNA779821). Scripts for analysis of data are available on request.

\section{Author contributions}

AG, AR, JCA and ID designed the study. JCA advised on the fear conditioning experiments. AG managed the project, carried out all stress procedures, behavioural experiments, dissections and RNA extractions. AR, JH and IS carried out sequencing of samples. AG and AR analysed the data and wrote initial drafts of the manuscript. JCA, ISM, ID provided advice on data analysis and interpretation. All authors contributed to the interpretation of the data, critically revised the manuscript, read and approved the final version before submission.

\section{Ethics approval and consent to participate}

All experiments were approved by the University of Sydney Animal Ethics Committee (2018/1425). All efforts were made to minimize animal suffering and to reduce the number of animals used.

\section{Competing interest statement}

I.W.D. manages a fee-for-service nanopore sequencing facility at the Garvan Institute of Medical Research that is a customer of Oxford Nanopore Technologies but has no further 
bioRxiv preprint doi: https://doi.org/10.1101/2021.11.25.468910; this version posted November 25, 2021. The copyright holder for this preprint (which was not certified by peer review) is the author/funder, who has granted bioRxiv a license to display the preprint in perpetuity. It is made available under aCC-BY-ND 4.0 International license.

financial relationship. The authors declare no other competing financial or non-financial interests. 


\section{References}

1. Maren, S. \& Holmes, A. Stress and Fear Extinction. Neuropsychopharmacology 41, 5879 (2016).

2. Johansen, Joshua P., Cain, Christopher K., Ostroff, Linnaea E. \& LeDoux, Joseph E. Molecular Mechanisms of Fear Learning and Memory. Cell 147, 509-524 (2011).

3. Zannas, A.S., Provençal, N. \& Binder, E.B. Epigenetics of Posttraumatic Stress Disorder: Current Evidence, Challenges, and Future Directions. Biological Psychiatry 78, 327-335 (2015).

4. Howie, H., Rijal, C.M. \& Ressler, K.J. A review of epigenetic contributions to posttraumatic stress disorder . Dialogues Clin Neurosci 21, 417-428 (2019).

5. Jonkhout, N., et al. The RNA modification landscape in human disease. Rna 23, 17541769 (2017).

6. Zhao, B.S., Roundtree, I.A. \& He, C. Post-transcriptional gene regulation by mRNA modifications. Nature Reviews Molecular Cell Biology 18, 31-42 (2017).

7. Livneh, I., Moshitch-Moshkovitz, S., Amariglio, N., Rechavi, G. \& Dominissini, D. The m(6)A epitranscriptome: transcriptome plasticity in brain development and function. Nat Rev Neurosci 21, 36-51 (2020).

8. Madugalle, S.U., Meyer, K., Wang, D.O. \& Bredy, T.W. RNA N(6)-Methyladenosine and the Regulation of RNA Localization and Function in the Brain. Trends Neurosci 43, 1011-1023 (2020).

9. Siegmund, A. \& Wotjak, C.T. A mouse model of posttraumatic stress disorder that distinguishes between conditioned and sensitised fear. J Psychiatr Res 41, 848-860 (2007).

10. Kao, C.Y., et al. Fluoxetine Treatment Rescues Energy Metabolism Pathway Alterations in a Posttraumatic Stress Disorder Mouse Model. Mol Neuropsychiatry 2, 46-59 (2016).

11. Assareh, N., et al. Cannabidiol disrupts conditioned fear expression and cannabidiolic acid reduces trauma-induced anxiety-related behaviour in mice. Behav Pharmacol 31, 591596 (2020).

12. Dedic, N., et al. Chronic $\mathrm{CRH}$ depletion from GABAergic, long-range projection neurons in the extended amygdala reduces dopamine release and increases anxiety. Nat Neurosci 21, 803-807 (2018).

13. Smith, K.L., et al. Microglial cell hyper-ramification and neuronal dendritic spine loss in the hippocampus and medial prefrontal cortex in a mouse model of PTSD. Brain Behav Immun 80, 889-899 (2019).

14. Brzozowska, N.I., et al. Genetic deletion of P-glycoprotein alters stress responsivity and increases depression-like behavior, social withdrawal and microglial activation in the hippocampus of female mice. Brain Behav Immun 65, 251-261 (2017). 
15. Christiansen, D.M. \& Berke, E.T. Gender- and Sex-Based Contributors to Sex Differences in PTSD. Curr Psychiatry Rep 22, 19 (2020).

16. Verbitsky, A., Dopfel, D. \& Zhang, N. Rodent models of post-traumatic stress disorder: behavioral assessment. Transl Psychiatry 10, 132 (2020).

17. ter Horst, J.P., Carobrez, A.P., van der Mark, M.H., de Kloet, E.R. \& Oitzl, M.S. Sex differences in fear memory and extinction of mice with forebrain-specific disruption of the mineralocorticoid receptor. European Journal of Neuroscience 36, 3096-3102 (2012).

18. Lin, H.C., et al. Promyelocytic leukemia zinc finger is involved in the formation of deep layer cortical neurons. J Biomed Sci 26, 30 (2019).

19. Gaber, Z.B., Butler, S.J. \& Novitch, B.G. PLZF regulates fibroblast growth factor responsiveness and maintenance of neural progenitors. PLoS Biol 11, e1001676 (2013).

20. Usui, N., et al. Zbtb16 regulates social cognitive behaviors and neocortical development. Transl Psychiatry 11, 242 (2021).

21. Cheng, H., Pablico, S.J., Lee, J., Chang, J.S. \& Yu, S. Zinc Finger Transcription Factor Zbtb16 Coordinates the Response to Energy Deficit in the Mouse Hypothalamus. Frontiers in Neuroscience 14 (2020).

22. Garcia-Calero, E. \& Puelles, L. Development of the mouse anterior amygdalar radial unit marked by Lhx9-expression. Brain Struct Funct 226, 575-600 (2021).

23. Zhuang, S., et al. Expression of Isl1 during mouse development. Gene Expr Patterns 13, 407-412 (2013).

24. von Huth, S., et al. Immunohistochemical Localization of Fibrinogen C Domain Containing 1 on Epithelial and Mucosal Surfaces in Human Tissues. J Histochem Cytochem 66, 85-97 (2018).

25. Lang, F., et al. (Patho)physiological significance of the serum- and glucocorticoidinducible kinase isoforms. Physiol Rev 86, 1151-1178 (2006).

26. Andero, R., Dias, B.G. \& Ressler, K.J. A role for Tac2, NkB, and Nk3 receptor in normal and dysregulated fear memory consolidation. Neuron 83, 444-454 (2014).

27. Lee, C.T., Ma, Y.L. \& Lee, E.H. Serum- and glucocorticoid-inducible kinase1 enhances contextual fear memory formation through down-regulation of the expression of Hes5. $J$ Neurochem 100, 1531-1542 (2007).

28. Licznerski, P., et al. Decreased SGK1 Expression and Function Contributes to Behavioral Deficits Induced by Traumatic Stress. PLOS Biology 13, e1002282 (2015).

29. Takeuchi, T., Duszkiewicz, A.J. \& Morris, R.G.M. The synaptic plasticity and memory hypothesis: encoding, storage and persistence. Philos Trans $R$ Soc Lond B Biol Sci 369, 20130288-20130288 (2013). 
30. Silva, A.J. Molecular and cellular cognitive studies of the role of synaptic plasticity in memory. Journal of Neurobiology 54, 224-237 (2003).

31. Luchkina, N.V. \& Bolshakov, V.Y. Mechanisms of fear learning and extinction: synaptic plasticity-fear memory connection. Psychopharmacology 236, 163-182 (2019).

32. Martin, S.J., Grimwood, P.D. \& Morris, R.G.M. Synaptic Plasticity and Memory: An Evaluation of the Hypothesis. Annual Review of Neuroscience 23, 649-711 (2000).

33. Marwha, D., Halari, M. \& Eliot, L. Meta-analysis reveals a lack of sexual dimorphism in human amygdala volume. Neurolmage 147, 282-294 (2017).

34. Blume, S.R., et al. Sex- and Estrus-Dependent Differences in Rat Basolateral Amygdala. J Neurosci 37, 10567-10586 (2017).

35. Morris, J.A., Jordan, C.L., King, Z.A., Northcutt, K.V. \& Breedlove, S.M. Sexual dimorphism and steroid responsiveness of the posterodorsal medial amygdala in adult mice. Brain Res 1190, 115-121 (2008).

36. Cooke, B.M. \& Woolley, C.S. Sexually dimorphic synaptic organization of the medial amygdala. J Neurosci 25, 10759-10767 (2005).

37. Andreano, J.M. \& Cahill, L. Sex influences on the neurobiology of learning and memory. Learn Mem 16, 248-266 (2009).

38. Daskalakis, N.P., Cohen, H., Cai, G., Buxbaum, J.D. \& Yehuda, R. Expression profiling associates blood and brain glucocorticoid receptor signaling with trauma-related individual differences in both sexes. Proc Natl Acad Sci U S A 111, 13529-13534 (2014).

39. Kim, G.S. \& Uddin, M. Sex-specific and shared expression profiles of vulnerability and resilience to trauma in brain and blood. Biol Sex Differ 11, 13 (2020).

40. Pooley, A.E., et al. Sex differences in the traumatic stress response: the role of adult gonadal hormones. Biol Sex Differ 9, 32 (2018).

41. Pooley, A.E., et al. Sex differences in the traumatic stress response: PTSD symptoms in women recapitulated in female rats. Biol Sex Differ 9, 31-31 (2018).

42. Issler, O., et al. Sex-Specific Role for the Long Non-coding RNA LINC00473 in Depression. Neuron 106, 912-926.e915 (2020).

43. Paden, W., et al. Sex differences in adult mood and in stress-induced transcriptional coherence across mesocorticolimbic circuitry. Translational Psychiatry 10, 59 (2020).

44. Girgenti, M.J., et al. Prefrontal cortex interneurons display dynamic sex-specific stressinduced transcriptomes. Translational psychiatry 9, 292-292 (2019).

45. Peña, C.J., et al. Early life stress alters transcriptomic patterning across reward circuitry in male and female mice. Nature Communications 10, 5098 (2019). 
46. Oikonomopoulos, S., et al. Methodologies for Transcript Profiling Using Long-Read Technologies. Frontiers in Genetics 11 (2020).

47. Wang, Y., Zhao, Y., Bollas, A., Wang, Y. \& Au, K.F. Nanopore sequencing technology, bioinformatics and applications. Nature Biotechnology 39, 1348-1365 (2021).

48. Amarasinghe, S.L., et al. Opportunities and challenges in long-read sequencing data analysis. Genome Biology 21, 30 (2020).

49. Umschweif, G., et al. Identification of Neurensin-2 as a novel modulator of emotional behavior. Molecular Psychiatry 26, 2872-2885 (2021).

50. Glatt, S.J., et al. Blood-based gene-expression predictors of PTSD risk and resilience among deployed marines: A pilot study. American Journal of Medical Genetics Part B: Neuropsychiatric Genetics 162, 313-326 (2013).

51. Pratanwanich, P.N., et al. Identification of differential RNA modifications from nanopore direct RNA sequencing with xPore. Nature Biotechnology (2021).

52. Leger, A., et al. RNA modifications detection by comparative Nanopore direct RNA sequencing. bioRxiv, 843136 (2019).

53. Meyer, Kate D., et al. Comprehensive Analysis of mRNA Methylation Reveals Enrichment in 3\&\#x2032; UTRs and near Stop Codons. Cell 149, 1635-1646 (2012).

54. Meyer, K.D., et al. 5' UTR m(6)A Promotes Cap-Independent Translation. Cell 163, 9991010 (2015).

55. Dominissini, D., et al. Topology of the human and mouse m6A RNA methylomes revealed by m6A-seq. Nature 485, 201-206 (2012).

56. Wang, K., Peng, J. \& Yi, C. The m6A Consensus Motif Provides a Paradigm of Epitranscriptomic Studies. Biochemistry (2021).

57. Xuan, J.-J., et al. RMBase v2.0: deciphering the map of RNA modifications from epitranscriptome sequencing data. Nucleic Acids Research 46, D327-D334 (2017).

58. Daniels, T.E., Olsen, E.M. \& Tyrka, A.R. Stress and Psychiatric Disorders: The Role of Mitochondria. Annual Review of Clinical Psychology 16, 165-186 (2020).

59. Manoli, I., et al. Mitochondria as key components of the stress response. Trends in Endocrinology \& Metabolism 18, 190-198 (2007).

60. Picard, M., McEwen, B.S., Epel, E.S. \& Sandi, C. An energetic view of stress: Focus on mitochondria. Front Neuroendocrinol 49, 72-85 (2018).

61. Zhang, L., et al. Mitochondria-focused gene expression profile reveals common pathways and CPT1B dysregulation in both rodent stress model and human subjects with PTSD. Translational Psychiatry 5, e580-e580 (2015). 
62. Flaquer, A., et al. Mitochondrial genetic variants identified to be associated with posttraumatic stress disorder. Translational psychiatry 5, e524-e524 (2015).

63. Naviaux, R.K. Metabolic features of the cell danger response. Mitochondrion 16, 7-17 (2014).

64. Kung, J.-C., Chen, T.-C., Shyu, B.-C., Hsiao, S. \& Huang, A.C.W. Anxiety- and depressivelike responses and c-fos activity in preproenkephalin knockout mice: Oversensitivity hypothesis of enkephalin deficit-induced posttraumatic stress disorder. Journal of Biomedical Science 17, 29 (2010).

65. Sah, R. \& Geracioti, T.D. Neuropeptide $Y$ and posttraumatic stress disorder. Molecular Psychiatry 18, 646-655 (2013).

66. Engel, M., et al. The Role of $\mathrm{m}(6) \mathrm{A} / \mathrm{m}$-RNA Methylation in Stress Response Regulation. Neuron 99, 389-403.e389 (2018).

67. Schaefer, M., Kapoor, U. \& Jantsch, M.F. Understanding RNA modifications: the promises and technological bottlenecks of the 'epitranscriptome'. Open Biology 7, 170077.

68. He, P.C. \& He, C. m(6) A RNA methylation: from mechanisms to therapeutic potential. Embo j 40, e105977 (2021).

69. Zhang, Z., et al. Single-base mapping of m(6)A by an antibody-independent method. Sci Adv 5, eaax0250 (2019).

70. Walters, B.J., et al. The Role of The RNA Demethylase FTO (Fat Mass and ObesityAssociated) and mRNA Methylation in Hippocampal Memory Formation. Neuropsychopharmacology 42, 1502-1510 (2017).

71. Widagdo, J., et al. Experience-Dependent Accumulation of N6-Methyladenosine in the Prefrontal Cortex Is Associated with Memory Processes in Mice. J Neurosci 36, 6771-6777 (2016).

72. Huang, Y., et al. Small-Molecule Targeting of Oncogenic FTO Demethylase in Acute Myeloid Leukemia. Cancer Cell 35, 677-691.e610 (2019).

73. Selberg, S., et al. Small-Molecule Inhibitors of the RNA M6A Demethylases FTO Potently Support the Survival of Dopamine Neurons. International Journal of Molecular Sciences 22 (2021).

74. Rauch, S., et al. Programmable RNA-Guided RNA Effector Proteins Built from Human Parts. Cell 178, 122-134.e112 (2019).

75. Rauch, S., He, C. \& Dickinson, B.C. Targeted m6A Reader Proteins To Study Epitranscriptomic Regulation of Single RNAs. Journal of the American Chemical Society 140, 11974-11981 (2018).

76. Buccitelli, C. \& Selbach, M. mRNAs, proteins and the emerging principles of gene expression control. Nat Rev Genet 21, 630-644 (2020). 
bioRxiv preprint doi: https://doi.org/10.1101/2021.11.25.468910; this version posted November 25, 2021. The copyright holder for this preprint (which was not certified by peer review) is the author/funder, who has granted bioRxiv a license to display the preprint in perpetuity. It is made available under aCC-BY-ND 4.0 International license.

77. Damase, T.R., et al. The Limitless Future of RNA Therapeutics. Frontiers in Bioengineering and Biotechnology 9 (2021). 


\section{Supplementary Material}

\section{Materials \& Methods}

\section{Animals \& Housing}

All experiments were conducted in accordance with the NHMRC Australian code for the care and use of animals for scientific purposes and approved by the Animal Ethics Committee of University of Sydney (AEC2018/1425). All efforts were made to minimise animal suffering and to reduce the number of animals used. Adult male and randomly cycling female C57BL/6J mice were obtained from the Animal Resources Centre (Western Australia) and housed in groups of 5 in individually ventilated cages (IVC) at the Brain \& Mind Centre (New South Wales) in a temperature/humidity-controlled environment $\left(21^{\circ} \mathrm{C}, 55.5 \%\right)$. Following 1 week of acclimatisation to reverse-phase lighting (lights ON: 21h00, OFF: 09h00), mice were singly housed in IVCs for an additional week. Food and water were available ad libitum.

\section{Cued Fear Conditioning \& Cued Fear Memory Testing}

The procedure for conditioning and testing of cued-fear memory is based on recently published work ${ }^{1-3}$. Briefly, mice were fear conditioned in a wooden sound-proof chamber, transparent front and rear-facing walls, opaque side walls with a white light and a metal grid floor (Context A). The conditioning session started with a $198 \mathrm{~s}$ acclimation period which was followed by a $30 \mathrm{~s} 80 \mathrm{~dB}$ tone $(9 \mathrm{KHz}$ sinewave, conditioned stimulus, $\mathrm{CS})$ that co-terminated with a $2 \mathrm{~s} 1.5 \mathrm{~mA}$ foot-shock delivered through the metal grid as a constant current (unconditioned stimulus, US). The conditioning session ended with a $60 \mathrm{~s}$ after-shock interval. Non-shock control mice were exposed only to the CS tone. Fear conditioning chambers were cleaned using $70 \%$ ethanol spray solution. Within 15 mins of being fear conditioned, one group of stress and control mice were culled, brains extracted and snap frozen. Cued fear memory was tested in another group of stress and control mice $24 \mathrm{hrs}$ later in a novel context (Context B: wooden sound-proof chamber, red light, red perspex flooring, peppermint oil scent). Following a 2 min baseline period, all mice were presented with $3 \times 30 \mathrm{sec}$ CS with an inter-tone interval of $1 \mathrm{~min}$. The memory retrieval session ended with a $60 \mathrm{~s}$ interval after the last tone was presented. Chambers were cleaned using F10 disinfectant spray solutions. Freezing was measured in response to the CS using the CleverSys FreezeScan ${ }^{\circledR}$ video $\operatorname{tracking}$ system and software.

\section{Tissue Collection \& RNA Extraction}

Mice were sacrificed by cervical decapitation within 15 min of being fear conditioned. Brains were extracted, snap-frozen in liquid nitrogen and stored at $-80^{\circ} \mathrm{C}$. As previously described ${ }^{4}$, bilateral punches ( 0.5 to $1 \mathrm{~mm}$ ) of the amygdala (AMG: central and basolateral amygdala) were made using the Paxinos and Watson Atlas as a guide ${ }^{5}$. Tissue was collected in RNA-free tubes and RNA was extracted using Qiagen RNeasy ${ }^{\mathrm{TM}}$ Micro kit (Qiagen, MD, USA) according to the manufacturer's instructions. RNA concentrations were quantified using a NanoDrop ${ }^{\mathrm{TM}}$ 
One spectrophotometer (Thermofisher Scientific ${ }^{\circledR}$, MA, USA) and only samples with $260 / 280$ ratios of greater than 1.7 were used for downstream analyses. Samples from 3-4 mice were randomly pooled for subsequent analyses by Illumina NextSeq and Promethion. mRNA was purified from total RNA pools using the Dynabeads ${ }^{\circledR}$ mRNA Purification Kit (Thermofisher Scientific $\left.{ }^{\circledR}, M A, U S A\right)$ according to the manufacturer's instructions.

\section{Illumina RNA sequencing}

Sequin RNA Mix (Hardwick et al. 2016) sequencing control was added at a fraction of $1 \%$ of total purified mRNA. Purified mRNA with Sequin was used to prepare libraries using the KAPA Stranded RNA-Seq Library Preparation Kit (Roche, CA, USA) with SeqCap Adapters (Roche CA, USA) as per manufacturer's instructions. The libraries were sequenced on an Illumina NextSeq 500 System, generating 24.6-48.9 million, 2 x 150bp read-pairs per sample.

\section{Nanopore direct-RNA sequencing}

Direct RNA sequencing was performed using the ONT Direct RNA Sequencing Kit (SQKRNA002), as per the manufacturer's instructions. $200 \mathrm{ng}$ of purified mRNA per sample was provided as input and optional first-strand cDNA synthesis was performed using SuperScript III Reverse Transcriptase (Thermo Fisher Scientific). For each sample, $30-50 \mathrm{ng}$ of prepped library was loaded onto a single ONT PromethION flow cell and sequenced for a maximum $\sim 72$ hours, generating 2.2-4.6 million native RNA reads per sample.

\section{Bioinformatics - Gene expression profiling}

Reads were trimmed using Trim Galore (Galaxy version 0.6.3) and subsequently aligned to the reference Mus musculus genome (GRCm38) using HISAT2 (Galaxy version 2.1.0). Reads were annotated (Gencode v.M25) and counted using featureCounts (Galaxy version 2.0.1). Biostatistics and visualization were run in $\mathrm{R}$ (version 3.6.3) with the Rstudio GUI (version 1.2.5033). For each comparison - male: stress(3) $v$ control(3), female:stress(3) v control(3) genes were filtered to have at least 5 reads in at least 2 samples for each gene and have gene biotype of protein-coding, long non-coding or microRNA. RUVSeq ${ }^{6}$ and DESeq ${ }^{7}$ were then used to quantify differential gene expression between groups. We set a threshold criteria of a minimum 0.2 expression fold-change and DGE FDR<0.05. Gene ontology and enrichment analysis was performed using EnrichR with a significance threshold set at FDR $<0.05^{8}$.

\section{qPCR Validation}

RNA was reverse-transcribed to complementary DNA using the Applied Biosystem ${ }^{\circledR}$ High Capacity cDNA Reverse Transcription Kit (10X RT Buffer, 25X dNTP mix (100mM), 10X RT Random Primers, Multiscribe ${ }^{\circledR}$ Reverse Transcriptase) on the Applied Biosystem ${ }^{\circledR}$ GeneAmp PCR System 9700 (Thermofisher ${ }^{\circledR}$, Waltham, MA, USA). qRT-PCR was carried out on the StepOnePlus ${ }^{\circledR}$ PCR machine (Thermofisher ${ }^{\circledR}$, Waltham, MA, USA) using the following primer assays designed by Integrated DNA Techologies (Skokie, Illinois, USA). Samples were heated to $95^{\circ} \mathrm{C} \times 10 \mathrm{~min}$, and then subjected to 40 cycles of amplification by melting at $95^{\circ} \mathrm{C} \times 15 \mathrm{~s}$ 
and annealing at $60^{\circ} \mathrm{C} \times 1 \mathrm{~min}$. Experimental samples were run in duplicates with $1.33 \mu \mathrm{L}$ complementary DNA (cDNA) per reaction. To check for amplicon contamination, each run also contained template free controls for each probe used. PCR data were normalized using $\beta$-actin and transformed using the $\Delta \Delta C$ t method.

\section{Bioinformatics - Epitranscriptome profiling}

To generate a comprehensive catalogue of high-confidence RNA modification sites present in the amygdala of male and female mice exposed to conditioned fear, we used two recently released software Nanocompore and Xpore on our direct RNA-seq libraries. Nanocompore collects current intensity and dwell time at each position, as the native RNA goes through the pore, and uses those variables to perform pairwise statistical comparisons between control and stress samples. The tool reports positions with a significant statistical difference for each of the individual variables, but also models a logistic regression that takes both current intensity and dwell time into account in search for significant differences. To minimise the false-positive discovery rate, we applied strict filtering to the results, only retaining putative modifications with a significant $p$-value (alpha $=0.01$ ) in all three statistical tests. Xpore, on the other hand, models the current intensity as a mixture of two Gaussian distributions, for modified and unmodified RNA, using prior information to help guide parameter estimation. Then given two conditions, such as control and stress, the tool is able to identify differential modification, but also quantitate them. We only selected putative modifications with significant differences in modification rates ( $t$-test, $p$-value $<0.01$ ) between the conditions and that were increasingly modified in the stress mice. To further increase the confidence in our dataset, we only retained modifications identified by both Nanocompore and Xpore, and that were at most $10 \mathrm{nt}$ apart from each other and we also collapsed modifications less than $10 \mathrm{nt}$ apart into a single modification site. We compared the coordinates of the modification sites identified using this approach against modification sites previously annotated in RMBase v2.0. We also performed gene set enrichment analysis using EnrichR with a significance threshold set at FDR $<0.05$. 


\section{References}

1. Assareh, N., et al. Cannabidiol disrupts conditioned fear expression and cannabidiolic acid reduces trauma-induced anxiety-related behaviour in mice. Behav Pharmacol 31, 591596 (2020).

2. Brzozowska, N.I., et al. Genetic deletion of P-glycoprotein alters stress responsivity and increases depression-like behavior, social withdrawal and microglial activation in the hippocampus of female mice. Brain Behav Immun 65, 251-261 (2017).

3. Smith, K.L., et al. Microglial cell hyper-ramification and neuronal dendritic spine loss in the hippocampus and medial prefrontal cortex in a mouse model of PTSD. Brain Behav Immun 80, 889-899 (2019).

4. Zapala, M.A., et al. Adult mouse brain gene expression patterns bear an embryologic imprint. Proceedings of the National Academy of Sciences of the United States of America 102, 10357 (2005).

5. Paxinos, G. The mouse brain in stereotaxic coordinates / George Paxinos, Keith Franklin (Academic, London, 2004).

6. Risso, D., Ngai, J., Speed, T.P. \& Dudoit, S. Normalization of RNA-seq data using factor analysis of control genes or samples. Nature Biotechnology 32, 896-902 (2014).

7. Anders, S. \& Huber, W. Differential expression analysis for sequence count data. Genome Biology 11, R106 (2010).

8. Xie, Z., et al. Gene Set Knowledge Discovery with Enrichr. Current Protocols 1, e90 (2021). 
a Density Mod. locations

b Density distribution of mods

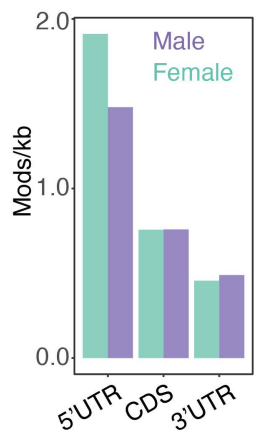

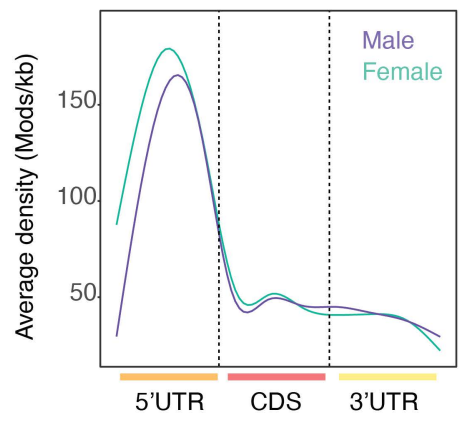

C RNA mod frequency per gene (Intersection)

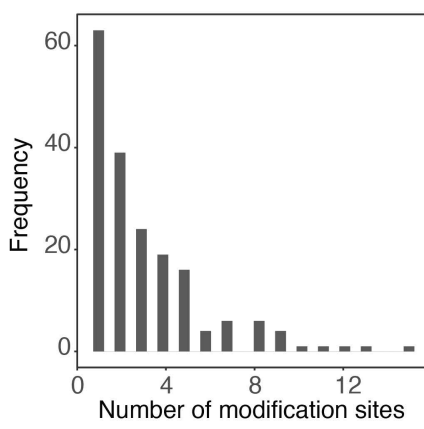

